\title{
Remarkable reversal of electrostatic interaction force on zwitterionic soft nanointerfaces in monovalent aqueous electrolyte: an AFM study at the single nanoparticle level
}

\author{
Audrey Beaussart ${ }^{1,2}$ * Céline Caillet $^{1,2}$, Isabelle Bihannic ${ }^{1,2}$, Ralf Zimmermann ${ }^{3}$, \\ and Jérôme F.L. Duval ${ }^{1,2 *}$
}

1

CNRS, LIEC (Laboratoire Interdisciplinaire des Environnements Continentaux), UMR7360, Vandoeuvre-lès-Nancy F-54501, France.

2 Université de Lorraine, LIEC, UMR7360, Vandoeuvre-lès-Nancy, F-54501, France.

3

Leibniz Institute of Polymer Research Dresden, Max Bergmann Center of Biomaterials Dresden,

Hohe Strasse 6, 01069 Dresden, Germany

* Corresponding authors:

A. Beaussart: audrey.beaussart@univ-lorraine.fr

J.F.L. Duval: jerome.duval@univ-lorraine.fr 


\section{TABLE OF CONTENTS ENTRY}

\section{Abstract}

Soft (nano)colloids are increasingly used in medical applications due to the versatile options they offer in terms of e.g. tunable chemical composition, adaptable physical properties and (bio)functionalization perspectives. Getting a clear understanding of the nature of the interaction forces that such particles experience with neighboring charged (bio)surfaces is a mandatory prerequisite to draw a comprehensive and mechanistic picture of their stability and reactivity, and to further optimize their current functionalities. In this study, adopting an original strategy for nanoparticle attachment to atomic force microscopy (AFM) tips, we demonstrate that the sign of electrostatic forces between carboxylate-terminated poly(amidoamine) nanodendrimers ( 9 $\mathrm{nm}$ in diameter) and planar cysteamine-coated gold surfaces can be tailored under fixed $\mathrm{pH}$ condition upon the sole variation of monovalent salt concentration in solution. The origin of this unconventional electrostatic force reversal is deciphered upon confrontation between AFM force measurements and mean-field force evaluation performed beyond the Derjaguin approximation with integrating dendrimer and cysteamine electrostatic properties derived independently from electrokinetic measurements. It is shown that the electrostatic force reversal (i) originates from the zwitterionic character of the nanodendrimer/solution interphase, and (ii) becomes operational under the strict condition that the sub-nanometric separation distance between peripheral carboxylate groups and intraparticulate amines is of the order of the characteristic electric Debye layer thickness. The possibility to mediate -via proper adjustment of monovalent salt content in solution- both the magnitude and sign of electrostatic forces acting on soft interfaces with zwitterionic functionality paves the way for 
the design of innovative strategies to control the stability of nanoparticles against aggregation, and to modulate their adhesion onto inorganic surfaces or living organisms.

\section{Introduction}

Electrostatic interactions play a key role in determining the stability of (bio)colloidal dispersions against aggregation. Whereas it is now accepted that the concept of zetapotential can be viewed as a relevant indicator to evaluate the stability of dispersions of hard particles that are impermeable to ions and fluid flow, recent theoretical and experimental studies have questioned its applicability to soft and core-shell particles, and planar surfaces that consist of- or are decorated by- 3D polyelectrolyte-like layers. ${ }^{1,2}$ In particular, the presence of such ion- and water-permeable polymeric structures leads to peculiar electrokinetic properties of these systems whose interpretation requires the use of formalisms that explicitly integrate the coupling between electrostatic and hydrodynamic fields developed under lateral flow conditions within and outside the charged polymeric layer compartment. ${ }^{1,2}$ A fundamental difference between soft and hard colloids is that the electrical double layer of the former is not restrictively located in the extraparticulate ionic environment, but rather resides for a part within the 3D particle body. As a result, the electrophoretic mobility of e.g. polymeric colloids, RNA bacteriophages or biopolymercoated bacteria reflects the structural features of an intraparticulate electrokinetically-active zone, the extension of which is fixed by the interplay between Debye length and characteristic flow penetration length scale. ${ }^{1}$ This remarkable feature has been exploited to unravel at the nanometric scale the structure of ionic-strength/pH-responsive thin polymer 
coatings, thermo-responsive thin films or surface-supported brushes with a spatial resolution that may overpass the performance of neutron-reflectivity based techniques. ${ }^{3-5}$

A particular attention has recently been paid to soft planar and particulate materials carrying anionic and cationic structural charges that are non-homogeneously distributed over the system body and/or at the interphase formed with the outer aqueous medium..$^{2,6-8}$ The electrokinetic response of these materials (e.g. electrophoretic mobility or streaming current for particles and planar surfaces, respectively) is indeed atypical as it undergoes an unconventional sign reversal upon variation of monovalent salt concentration in bulk solution. $^{2,6-8}$ The basis of such remarkable sign reversal stems from the zwitterionic organization of the cationic and anionic functional groups within the electrokinetically active zone of the soft substrate/medium interphase probed by the pressure-driven flow (streaming current) or electroosmotic flow (electrophoresis). The $\mathrm{pH}$ and salinity conditions where sign reversal occurs were shown to be determined by the ratio between density numbers of anionic and cationic structural charges, their typical separation distance, the electrostatic Debye length and the hydrodynamic Brinkman length scale. ${ }^{2,6-10}$ In turn, the respective contributions of e.g. peripheral anionic and internal cationic structural charges to the surface potential of a soft zwitterionic material depend on how far electric double layer probes their spatial organization within the material body. The here-described phenomenon is not to be confused with charge reversal of colloids (also called overcharging) in solution containing multivalent ions. ${ }^{11-13}$ The experimental and theoretical evidence for reversal of soft particle electrophoretic mobility in monovalent electrolyte solution has been recently reported for carboxylate-terminated poly(amidoamine) (PAMAM-COOH) dendrimers $(\sim 4 \mathrm{~nm}$ in radius) that possess protonable tertiary amine groups located at the intraparticulate branching points, and dissociable peripheral carboxylic groups. ${ }^{14}$ More generally, dendrimers 
are synthetic hyperbranched nanostructures composed of repeated branching cycles forming a core and a dense outer shell with functional groups that can be chemically modified. Their use has been proposed in various fields such as catalysis, material science or in biology/medicine, where they serve, among others, as imaging agents, antimicrobial compounds or drug carriers. ${ }^{15-18}$

In view of the above arguments, it is expected that the electrostatic interaction force between a given surface and a zwitterionic nanoparticle (e.g. a PAMAM-COOH dendrimer) both carrying surface charges of the same sign, should change from repulsive to attractive with changing the concentration of monovalent electrolyte in bulk solution. Unfortunately, experimental evidence for such a non-conventional electrostatic switch is still lacking to date. The reason stems from the technical difficulty to probe electrostatic forces between a surface and an individual nanoparticle under conditions where the thickness of the electric double layer (ca. $10 \mathrm{~nm}$ to $1 \mathrm{~nm}$ in $1 \mathrm{mM}$ to $100 \mathrm{mM}$ monovalent electrolyte concentration) is comparable to particle size. Atomic force microscopy (AFM) is a versatile technique for measuring forces between interacting entities with a piconewton resolution. While AFM has already been used to probe the interaction between dendrimers-decorated micronic silica ${ }^{19}$ or latex particles, ${ }^{20,21}$ its use to measure electrostatic force acting on a single particle with nanometric dimension has, to the best of our knowledge, never been reported.

Here, we evidence for the first time that electrostatic interaction force between PAMAM$\mathrm{COOH}$ nanodendrimer (G8.5 generation with negatively charged surface and positively charged core, Fig. 1a) and a positively charged cysteamine-coated gold surface changes sign with modulating the concentration of monovalent salt in solution. For that purpose, an original strategy is developed to covalently graft the dendrimers to an AFM tip (20 nm 
hemispherical apex), and the forces acting on the particle located at the apex of the tip are measured upon approach to the cysteamine-coated gold surface over a broad range of $\mathrm{KNO}_{3}$ electrolyte concentrations ( $1 \mathrm{mM}-200 \mathrm{mM}$ ) at different solution $\mathrm{pH}$ values (3.5 to 6.5) (Fig. 1b). At fixed $\mathrm{pH}$, it is shown that the threshold salt concentration value marking the transition between repulsion and attraction regimes remarkably coincides with that where reversal of dendrimer electrophoretic mobility is measured. This finding evidences that the force reversal originates from the counterintuitive salt concentration-mediated contributions of intraparticulate amino and peripheral carboxylate charges to the electrostatic potential distribution operational at the zwitterionic dendrimer/medium nanointerphase. Quantitatively, the electrostatic force-separation distance curves were successfully reconstructed from mean-field evaluation at the non-linear Poisson-Boltzmann (PB) level beyond the here-inapplicable conventional Derjaguin approximation. To that end, the dendrimer and cysteamine electrostatic surface features retrieved from independent electrophoresis and streaming current measurements, respectively, were used to properly define the electrostatic boundaries required to solve the governing PB equation. Overall, this work highlights a new efficient way to switch the sign of electrostatic forces acting on (nano)colloids in monovalent electrolyte solution upon tailoring the zwitterionic character of their nanointerphase formed with the outer electrolytic medium.

\section{Results and discussion}

Electrophoretic mobility reversal of G8.5 PAMAM-COOH dendrimer with varying concentration of background monovalent electrolyte. 
The current section investigates the electrokinetic behavior of PAMAM-COOH dendrimers - soft nanoparticles consisting of a hyperbranched amino-core which possesses protonable tertiary amine groups, and a peripheral shell made of dissociable carboxylic groups - in a monovalent electrolyte. Fig. 2a displays the electrophoretic mobility $\mu$ of G8.5 PAMAM$\mathrm{COOH}$ dendrimers as a function of $\mathrm{KNO}_{3}$ electrolyte concentration $c^{\infty}$ at various solution $\mathrm{pH}$ values ranging from 3.5 to 6.5 . Under the extreme 3.5 and $6.5 \mathrm{pH}$ conditions, $|\mu|$ decreases while increasing $c^{\infty}$ due to significant screening of the dendrimer charges by electrolyte ions. At $\mathrm{pH}=3.5, \mu$ remains positive regardless of salt concentration, indicating that dendrimer mobility is predominantly determined by intraparticulate protonated tertiary amine groups (with dissociation $\mathrm{p} K_{1}$ value). Conversely, for $\mathrm{pH}$ values exceeding 6 , the contribution of the strongly dissociated carboxylic groups (dissociation $\mathrm{p} K_{2}$ value) located in the peripheral dendrimer shell dominates and leads to negative electrophoretic mobility $\mu$. For given $\mathrm{pH}$ value in the intermediate 4-5.1 range, a striking mobility reversal is observed with increasing $c^{\infty}$ and the $\mathrm{pH}$ value marking a zero dendrimer electrophoretic mobility (the so-called Point of Zero Mobility, PZM in short) obviously decreases with increasing $c^{\infty}$. The latter trend is refined in Fig. 3a where the set of $\mathrm{pH}$ and $c^{\infty}$ conditions in line with zero dendrimer mobility is collected after systematic measurement of the electrokinetic dendrimer response at $\mathrm{KNO}_{3}$ concentrations finely tuned in the $1 \mathrm{mM}$ to $300 \mathrm{mM}$ range.

The above unconventional electrokinetic behavior basically originates from the extent by which the electric double layer penetrates within the particle body. ${ }^{2}$ In detail, with increasing the content of monovalent salt in solution, charge screening leads to an electric double layer that increasingly recedes within the intraparticulate peripheral dendrimer region. ${ }^{14}$ As a result, dendrimer electrophoretic mobility significantly reflects the negative 
charges stemming from the dissociation of the carboxylic groups provided that solution $\mathrm{pH}$ is significantly larger than $\mathrm{p} K_{2}$. Conversely, at sufficiently low electrolyte concentrations, the contribution of the carboxylic surface layer to dendrimer mobility becomes less significant in defining the potential distribution across the particle/medium interphase as the double layer extends deep inside the particle body. Consequently, under such salinity conditions the electrophoretic mobility becomes predominantly governed by the only electrostatic features of the intraparticulate amino-core component and the more so as $\mathrm{pH}$ is well below the dissociation value $\mathrm{p} K_{1}$ of the tertiary amine groups. Stated differently, the lower the salt concentration, the more significant is the contribution of the particulate core region to the measured dendrimer electrophoretic mobility. In the hypothetical situation where amino and carboxylate charges would be homogeneously mixed within the particle volume, the PZM would be independent of $c^{\infty}$ and the electrokinetic response typical of that expected for amphoteric materials. ${ }^{2}$ When introducing zwitterionicity, the dependence of the PZM on $c^{\infty}$ appears as a result of the respective $c^{\infty}$-mediated contributions of the cationic and anionic dendrimer regions to particle interfacial electrostatics and electrokinetics. The steepest change in PZM with varying $c^{\infty}$ is observed in the $30 \mathrm{mM}-150 \mathrm{mM}$ range (Fig. 3a), which corresponds to $c a .1 .8 \mathrm{~nm}$ to $0.8 \mathrm{~nm}$ electric Debye layer thickness. This range of Debye lengths is that where the carboxylate surface layer plays a significant role in determining interfacial dendrimer electrostatics and electrokinetics. It satisfactorily compares with the reported $0.5 \mathrm{~nm}-0.6 \mathrm{~nm}$ distance separating the outermost amino layer of the particle core from the peripheral carboxylic surface layer. ${ }^{22}$

The dendrimer electrokinetic properties reported in Figs. $2 a$ and $3 a$ are in further quantitative agreement with predictions from electrohydrodynamic formalism previously 
reported by the authors for soft chemically-stratified particles and successfully applied to G6.5 PAMAM-COOH dendrimers. ${ }^{14}$ Briefly, first assuming that anionic and cationic dendrimer charges are homogeneously distributed according to step-function like profiles in the radial dimension $r$ (Fig. 1a) the dependence of dendrimer mobility on $\mathrm{pH}$ and electrolyte concentration can be consistently reproduced only at sufficiently large salt concentrations $c^{\infty}$, upon adjustment of the dissociation $\mathrm{p} K$ values pertaining to amine and carboxylic groups ( $\mathrm{p} K_{1}$ and $\mathrm{p} K_{2}$, respectively) and of the density of carboxylic functional groups in the dendrimer periphery (dotted lines in Fig. 2a). ${ }^{14}$ The theoretical predictions overestimate the absolute values of the magnitude of dendrimer mobility in the low electrolyte concentration regime. As extensively discussed by Moussa et al., ${ }^{14}$ the discrepancy is due to welldocumented increase of dendrimer size at low $c^{\infty}$ as a result of coulombic repulsion between neighboring intraparticulate amines at $\mathrm{pH}<\mathrm{p} K_{1}$ or between surface carboxylic groups at $\mathrm{pH}>\mathrm{pK}_{2}{ }^{23}$ This change in dendrimer size is associated with an increase of the interfacial length scale, denoted as $\alpha_{\mathrm{H}}$, over which friction on electroosmostic flow is operational. In turn, it leads to a reduction of the mobility (in absolute value) compared to the situation where dendrimer size is considered independent of $\mathrm{pH}$ and $c^{\infty}$ (dotted lines in Fig. 2a). This process is very well captured by theory upon increasing $\alpha_{\mathrm{H}}$ with decreasing $c^{\infty}$, which reflects the expansion of the dendrimer structure to the outer electrolyte solution (solid lines in Fig. 2a). The reader is referred to Ref. ${ }^{14}$ for further details. The dendrimer surface potential $\psi_{\mathrm{s}}$ derived from the aforementioned analysis of the electrokinetic features of PAMAM-COOH dendrimer particles are reported in Fig. $2 b$ as a function of salt concentration at different $\mathrm{pH}$ values. The PZM coincides with the $\mathrm{pH}$ value where the surface potential $\psi_{\mathrm{s}}$ is zero. 
Streaming current properties of cysteamine-coated gold planar surfaces.

In view of assessing the electrostatic interactions between a PAMAM-COOH dendrimer and a positively-charged surface, the zeta $(\zeta)$-potential of a cysteamine-coated gold substrate was derived from streaming current measurements performed under different salt and $\mathrm{pH}$ conditions using an electrokinetic microslit set-up detailed elsewhere. ${ }^{24}$ Briefly, the streaming current arises from the displacement of the net counter charge under action of an applied pressure gradient $\Delta P$ in the direction parallel to two cysteamine-coated surfaces mounted in parallel in the microslit cell (Fig. 1c). Neglecting contribution from electronic surface charge density of the supporting gold surfaces, the measured streaming current was converted here to $\zeta$-potential value using the Smoluchowski equation

$$
\frac{I_{\mathrm{str}}}{\Delta P}=\frac{S \varepsilon \zeta}{\eta L}
$$

, where $L$ is the channel length, $S$ the cross-section area of the streaming channel, $\eta$ the dynamic fluid viscosity and $\varepsilon$ the dielectric permittivity of the medium (Fig. 1c). Typical values of $I_{\text {str }} / \Delta P$ and $\zeta$ are reported in Fig. $2 \mathrm{c}$ as a function of $c^{\infty}$ at $\mathrm{pH} 3.5$ and 5.1. As expectedly, they confirm the positive surface charge carried by cysteamine-coated gold surfaces in particular at $\mathrm{pH} 5.1$ and in the $15 \mathrm{mM}-35 \mathrm{mM}$ range that corresponds to conditions where -within experimental error- mobility reversal of PAMAM-COOH dendrimer takes place (Figs. 2a,b).

Reversal of electrostatic interaction force between PAMAM-COOH dendrimer and cysteamine-coated gold planar surface. 
Following an original strategy based on amino-carboxyl linking chemistry (see details in Methods section), PAMAM- $\mathrm{COOH}$ dendrimers were attached to AFM silicon-nitride tips. Briefly, the tips were first functionalized with amino groups, and subsequently immersed in a PAMAM-COOH dendrimer dispersion partially activated with NHS/EDC (Nhydroxysuccinimide/ethyl(dimethylaminopropylcarbodiimide). This procedure results in the covalent attachment of PAMAM dendrimers to the tip. Force measurements were then performed between the dendrimer-functionalized tip and a cysteamine self-assembled monolayer surface (Fig. $1 \mathrm{~b}$ ) at various $\mathrm{KNO}_{3}$ electrolyte concentrations and $\mathrm{pH}$ values below and above the corresponding Point of Zero Mobility (Fig. 3a). The confrontation between theory and force measurements collected at sufficiently high electrolyte concentration (see details below) supports the presence of a single dendrimer nanoparticle at the very tip apex. Representative approaching force-distance curves are reported in Fig. $3 \mathrm{~b}$ at $\mathrm{pH} 4.7$ and $c^{\infty}=$ $2 \mathrm{mM}, 10 \mathrm{mM}, 30 \mathrm{mM}$ and $200 \mathrm{mM}$. At $2 \mathrm{mM} \mathrm{KNO}_{3}$ concentration, i.e. the situation where pH 4.7 is well below the PZM of about 5.6 (Fig. 3a), the force gradually increases as the distance between the dendrimer-coated tip and the cysteamine substrate decreases. This repulsive force arises from the interactions between overlapping electric double layers extending from the dendrimer particle and the planar substrate with both positive surface potentials (as demonstrated from analysis of their respective electrokinetic features, see Figs. $2 b, c)$. As the salt concentration increases from $2 \mathrm{mM}$ to $10 \mathrm{mM}$, the magnitude of the repulsive force decreases and this force becomes practically undetectable at $30 \mathrm{mM}$. It is emphasized that the $30 \mathrm{mM}$ and $\mathrm{pH} 4.7$ conditions are those corresponding to the onset of sign reversal of the effective surface potential of the PAMAM-COOH dendrimer (Fig. 2b) in line with the quasi zero mobility measured under such conditions $\left(\mu<0.5 \times 10^{-8} \mathrm{~m}^{2} \mathrm{~V}^{-1} \mathrm{~s}^{-1}\right.$, Fig. 2a). Interestingly, at $200 \mathrm{mM} \mathrm{KNO}_{3}$ concentration, while the potential of the cysteamine 
surface remains positive, the force-distance profile pinpoints an attraction of the tip to the substrate (Fig. 3b). This is explained by the now negative surface potential of the attached dendrimer particle under such salinity condition (Fig. 2b). The trends displayed in Fig. 3b are maintained when recording several force-separation distance curves at various spots on the planar surface, which is illustrated in Fig. $4 \mathrm{~b}$ representing the occurrence frequency of attractive and repulsive events as a function of electrolyte concentration. Obviously, the detection of attractive events at sufficiently large electrolyte concentrations is rendered difficult by particle/substrate charge screening and associated reduction in the magnitude of the measured force. At the extreme $\mathrm{pH}$ values 3.5 and 6.5, i.e. situations where the sign of the electrophoretic mobility and surface potential of the dendrimer particles remain positive and negative over the whole range of tested salinity conditions, respectively, the dendrimercoated tip is always repelled from the cysteamine surface at pH 3.5 (Fig. 4a) and attracted to the cysteamine substrate at $\mathrm{pH} 6.5$ (Fig. 4c). This elegant agreement between the remarkable electrostatic features of dendrimer particles revealed by electrokinetics and by AFM force measurements demonstrate that the adopted grafting procedure does not impact on the surface charge of the dendrimer. In addition, it evidences that modulation of the sign of the electrostatic force is possible upon sole adjustment of the concentration in monovalent electrolyte. This force sign reversal is inherently attributed to the zwitterionic functionality of the dendrimer/solution interphase.

\section{Confrontation between AFM force-separation distance measurements and theoretical mean-field force evaluation.}


For sufficiently low pH values compared to the PZM, the AFM tip is always subjected to repulsion forces at separation distance $D$ lower than $c a .50 \mathrm{~nm}$ (Fig. 5a). Under such conditions, it is found that the repulsive force decreases with $D$ according to the classical exponential expression

$$
F_{\mathrm{el}}=A \exp (-\kappa D)
$$

, where $F_{\mathrm{el}}$ is the measured electrostatic force and $A$ is the force at zero separation distance. Experimental values of the decay length $\kappa^{-1}$ obtained at different electrolyte concentrations and $\mathrm{pH} 3.5$ are collected in Fig. 5b and are -within experimental error- in good agreement with the Debye length derived from $\kappa^{-1}=\sqrt{R T \varepsilon /\left(2 F^{2} c^{\infty}\right)}$ where $F, R$ and $T$ are the Faraday constant, the gas constant and the temperature, respectively. Equation 2 is typical for cases where electrostatic potentials prevailing in the overlapping electric double layers region are sufficiently low in magnitude to legitimate applicability of the Debye-Hückel approximation. ${ }^{25}$ At $\mathrm{pH} 6.5$, most of the force-distance curves underpin attraction between dendrimer and cysteamine-coated substrate (Fig. 5c). The shape of these force-distance curves at short separation distance consists of a linear section with few experimental points (Fig. 5c, insert), which indicates a fast 'snap-in' of the tip to the planar surface. This jump occurs when the attraction exceeds the maximum attractive force measurable by the cantilever ${ }^{26}$ and, consequently, it cannot be quantitatively interpreted on the basis of eq. 2 . Confirmation that the attraction between the dendrimer-decorated tip and the SAM surface originates from electrostatics is supported by the reduction of force (taken in absolute value) with increasing salt concentration in solution (Fig. 5d).

Given the aforementioned experimental bias encountered for situations where substrate-dendrimer attraction sets in, further quantitative interpretation is restricted to the 
only conditions where repulsion is operational. Experimental values of the prefactor $A$ involved in eq 2 are reported for $\mathrm{pH} 3.5$ in Fig. 5e (symbols) in the $1 \mathrm{mM}-200 \mathrm{mM}$ range of electrolyte concentrations where dendrimer-substrate repulsion is measured (Fig. 4). We attempted to evaluate quantitatively the electrostatic interaction force (or equivalently the prefactor $A$ in eq. 2) obtained from AFM analysis on the basis of the Surface Element Integration (SEI) technique proposed by Bhattachrjee et al. ${ }^{27}$ Briefly, the method allows the evaluation of the searched interaction force from the corresponding interaction energy per unit surface area between two infinite flat plates. Unlike the common Derjaguin's integration framework, ${ }^{28} \mathrm{SEI}$ is applicable to particles of any size and is not solely restricted to large particles (i.e. with a radius larger than $\kappa^{-1}$ ) for which curvature effects on electrostatic potential distribution are insignificant. ${ }^{27}$ Under the salinity conditions adopted in this work, the assumptions underlying the validity of the approximate Derjaguin's method are not applicable as the dendrimer radius $d$ may be of the order of magnitude of the electric double layer thickness $\kappa^{-1}$ (thick double layer regime).

Following the SEI procedure introduced above, ${ }^{27}$ the electrostatic interaction energy $E_{\mathrm{s}-\mathrm{p}}$ (in J) of any spherical particle of radius $R_{\text {eff }}$ and a planar substrate (subscripts ' $\mathrm{s}$ ' and ' $\mathrm{p}$ ', respectively) separated by a distance $D$ (Fig. 1b) may be written after some arrangements in the form $E_{\mathrm{s}-\mathrm{p}}(D)=\frac{2 \pi C}{\kappa^{3}} \int_{0}^{\kappa R_{\mathrm{eff}}} \bar{E}_{\mathrm{p}-\mathrm{p}}\left(\kappa D+\kappa R_{\mathrm{eff}}\left(1-\sqrt{1-\left(\frac{\xi}{\kappa R_{\mathrm{eff}}}\right)^{2}}\right)\right)-\bar{E}_{\mathrm{p}-\mathrm{p}}\left(\kappa D+\kappa R_{\mathrm{eff}}\left(1+\sqrt{1-\left(\frac{\xi}{\kappa R_{\mathrm{eff}}}\right)^{2}}\right) \xi \mathrm{d} \xi\right.$

, with $C=\kappa^{2} \varepsilon(R T / F)^{2} / 2\left(\mathrm{~N} \mathrm{~m}^{-2}\right)$, and $\xi$ is a dummy integration variable. In eq. $3, \bar{E}_{\mathrm{p}-\mathrm{p}}$ is the dimensionless electrostatic interaction energy between two infinite flat plates i.e. with 
ignoring dendritic particle curvature. $\bar{E}_{\mathrm{p}-\mathrm{p}}$ at a separation distance $D$ between such plates is defined from proper integration of the corresponding (dimensionless) disjoining pressure $\bar{\Pi}_{\mathrm{p}-\mathrm{p}}$ according to

$$
\bar{E}_{\mathrm{p}-\mathrm{p}}(D)=\int_{\kappa D}^{\infty} \bar{\Pi}_{\mathrm{p}-\mathrm{p}}(\xi) \mathrm{d} \xi
$$

, where $\bar{\Pi}_{\mathrm{p}-\mathrm{p}}$ at a given plate-to-plate separation distance $D$ is defined by an osmotic and Maxwell stress contribution according to

$$
\bar{\Pi}_{\mathrm{p}-\mathrm{p}}(D)=2\left[\cosh \left(y\left(X=X^{*}\right)\right)-1\right]-(\kappa D)^{-2}\left(\frac{\mathrm{d} y(X)}{\mathrm{d} X}\right)_{X=X^{*}}^{2}
$$

, where we introduced the dimensionless position variable $X=x / D$ with $x$ running from 0 to $D$ (Fig. 1b). It is stressed that in virtue of the principle of mechanical equilibrium for the interacting surfaces at fixed $D, \bar{\Pi}_{\mathrm{p}-\mathrm{p}}(D)$ may be evaluated for any value of $X^{*}$ across the overlapped electric double layers with $0 \leq X^{*} \leq 1$. In eq. 5 , the dimensionless electrostatic potential distribution $y(X)=F \psi(X) /(R T)$ between the interacting plates separated by $D$ follows from integration of the non-linear Poisson Boltzmann equation

$$
\frac{\mathrm{d}^{2} y(X)}{\mathrm{d} X^{2}}-(\kappa D)^{2} \sinh (y(X))=0
$$

, with the boundaries $y(X=0)=y_{\mathrm{p}}$ and $y(X=1)=y_{\mathrm{s}}$ where $y_{\mathrm{p}}$ and $y_{\mathrm{s}}$ are - in our case the dimensionless surface potentials of the SAM surface and of the dendrimer particle as derived from analyses of streaming current and electrophoresis measurements, respectively. Finally, the sought interaction force $F_{\mathrm{s}-\mathrm{p}}(D)$ between the SAM surface and the particle separated by the distance $D$ simply follows from derivation of $E_{\mathrm{s}-\mathrm{p}}(D)$ via 


$$
F_{\mathrm{s}-\mathrm{p}}(D)=-\frac{d E_{\mathrm{s}-\mathrm{p}}(D)}{\mathrm{d} D}
$$

, where $E_{\mathrm{s}-\mathrm{p}}(D)$ is defined by eq. 3. For a given separation distance $D$, the potential distribution $y(X)$ was obtained from the numerical solution of eq. 6 according to collocation method. ${ }^{29}$ Solutions were iteratively obtained for $D$ values ranging from $\kappa D>>1$ where SAM surface and dendrimer particle are electrically isolated down to $\kappa D=2$. Once the potential distribution is determined, the disjoining pressure $\bar{\Pi}_{p-p}(D)$ could be easily evaluated as a function of $\kappa D$ (eq. 5), and the integrations following eq. 3-4 were performed by means of Simpson's method and the derivative in eq. 7 evaluated with use of Ridder's method of polymomial extrapolation. ${ }^{30}$ The validity of the numerical scheme (written in FORTRAN) was addressed by comparing results with those reported in Ref ${ }^{27}$ (Fig. 5 therein). Under the specific $\mathrm{pH}$ and salinity conditions adopted in this work, we verified that eq. 7 systematically leads to the classical exponential form (eq. 2) over the range of separation distances $D$ in line with $\kappa D>2$.

Given the here-applicable situation where particles are grafted onto an hemispherical AFM tip, theoretical values of $A$ can only be estimated after properly defining the effective radius $R_{\text {eff }}$ of the spherical entity interacting with the planar SAM surface. Let's consider the case where the interaction takes place at sufficiently low electrolyte concentrations such that the inequality $\kappa d \leq 1$ is verified. Then, the equipotential surfaces (dotted lines in Fig. $5 f$ ) follow the contour of the ensemble AFM tip plus grafted dendrimers, i.e. a sphere with effective radius $R_{\text {eff }}=19 \mathrm{~nm}$. Conversely, at high salinity in line with the condition $\kappa d>>1$, the equipotential surfaces strictly follow the contour of the dendrimer particles and therefore the effective radius $R_{\text {eff }}$ of the interacting sphere identifies with that of a single dendrimer, i.e. $R_{\mathrm{eff}}=d=4.5 \mathrm{~nm}$ (Fig. $5 \mathrm{f}$, right). Theoretical values of $A$ were derived at $\mathrm{pH}$ 
3.5 within the framework of the electrostatic limits defined above (Fig. 5e) corresponding to thick and thin electric double layer regimes. For that purpose, the required particle surface potential $y_{\mathrm{s}}$ was directly inferred from dendrimer electrophoretic mobility analysis (Fig. 2b) and the surface potential $y_{\mathrm{p}}$ of the planar substrate from streaming current data (Fig. 2c) with further adopting $y_{\mathrm{p}}=1.1$ and $y_{\mathrm{p}}=1$ at $100 \mathrm{mM}$ and $200 \mathrm{mM}$ salt concentration, respectively. Figure 5 e shows the excellent agreement between measured and theoretical value of $A$ as obtained at low salinity $(1 \mathrm{mM})$ without adjustment of any parameter under such salt concentration condition, and the proper reconstruction of the dependence of $A$ on salt concentration in the thin electric double layer limit $\left(c^{\infty}>30 \mathrm{mM}\right)$. Altogether, the theoretical analysis confirms the electrostatic nature of the measured interaction force and further supports the origin of the unconventional force reversal with changing monovalent salt concentration.

\section{Conclusions.}

Controlling the stability of dispersions of soft nanoparticles requires a clear understanding of the electrostatic interactions they experience when in the vicinity of charged particulate or planar interfaces. In this study, electrokinetic and atomic force microscopy experiments are combined to assess on a quantitative basis the electrostatic interaction force between charge-layered nanoparticles of the PAMAM-COOH G8.5 dendrimer type (4.5 $\mathrm{nm}$ in radius) and a planar cysteamine-coated gold surface. The technically challenging AFM force measurements performed at the nanoparticle level are made possible by the here-adopted NHC-EDC amino-carboxyl-linking chemistry for grafting PAMAM-COOH nanodendrimers at the apex of amino-activated AFM tips with $10 \mathrm{~nm}$ curvature radius. Supported by advanced 
soft particle electrokinetic analysis and by electrostatic force evaluation beyond the conventional and approximate Derjaguin approximation, measurements highlight the possibility to (i) switch the sign of the surface potential of dendrimer nanoparticles with varying monovalent electrolyte concentration in solution, and (ii) therefore tune the sign of the electrostatic force dendrimer particles feel when approaching a charged surface. It is further demonstrated that the physical basis of these remarkable findings is intimately tied to the zwitterionic functionality of PAMAM-COOH dendrimers. Depending on the extension of the electric double layer within the particle body, the dendrimer surface potential reflects indeed the dissociation features of amines distributed within the internal particle core, those of the peripheral carboxylic groups or is determined by both amino- and surface carboxylicmediated contributions. The results have far-reaching implications in terms of monitoring electrostatic interaction forces between soft nanoparticles and charged (bio)environments. In particular, both force magnitude and sign may be tailored from proper chemical modifications of nanoparticles leading to a controlled separation distance between oppositely charged functional groups within the particle body and/or the subsurface particulate region. Following such an approach, the degree of tunability of the interaction forces is directly controlled by the zwitterionic nanoparticle features effectively tailored with changing monovalent electrolyte concentration in solution. The here-evidenced results could serve as a basis for refining current bio-nanotechnological and nanotherapeutic strategies aimed at e.g. transporting drugs carried by nanoparticles to specific cancer cells (targeted cell lysis) or to infected plant tissues (targeted nano-remediation). 


\section{Methods.}

Carboxylated PAMAM Dendrimers. Half-generation G8.5 carboxylate-terminated poly(amidoamine) (PAMAM) dendrimers dispersed in water were purchased from Dendritech (Dendritech Inc, U.S.A.). They consist of a hyperbranched amino-core which possesses protonable tertiary amine groups and a peripheral shell made of dissociable carboxylic groups. ${ }^{14,22,31}$ Each dendrimer contains $2\left(2^{n+1}-1\right)$ tertiary amine groups and $2^{n+2}$ carboxylic groups, ${ }^{14} n$ being the dendrimer generation with $n=9$ for G8.5 dendrimer generation adopted in this work (i.e. 2046 tertiary amine and 2048 carboxylic groups).

Electrophoresis. The electrophoretic mobility of G8.5 PAMAM-COOH dendrimers was measured as a function of $\mathrm{pH}$ and $\mathrm{KNO}_{3}$ (Sigma-Aldrich, purity > 99\%) concentration at room temperature using a Zetasizer Nano ZS instrument (Malvern Instruments). $\mathrm{pH}$ values were adjusted by proper addition of $\mathrm{HNO}_{3}(0.1 \mathrm{M}$, Sigma, purum p.a.) and $\mathrm{KOH}(0.1 \mathrm{M}$, VWR, convol Normadose) solutions. Each reported data point is the average of at least 3 distinct mobility acquisitions. Particle size was measured over the whole range of $\mathrm{pH}$ and salinity conditions tested in this work using the above Zetasizer equipement. Within the experimental uncertainty, we found $d=9 \pm 1 \mathrm{~nm}$ for G8.5 dendrimer diameter with no sign of aggregation even under extreme electrolyte concentration conditions (300 mM $\mathrm{KNO}_{3}$ concentration in this work).

Streaming current measurements. To perform the electrokinetic experiments on the cysteamine surface, clean glass blocks $(20 \mathrm{~mm} \times 10 \mathrm{~mm})$ were first coated with a $5 \mathrm{~nm}$-thick chromium ( $\mathrm{Cr}$ ) layer followed by a $30 \mathrm{~nm}$-thick layer of gold ( $\mathrm{Au}$ ) using an electron-beam thermal evaporator. Gold substrate were then immersed for 1.5 hour in a $20 \mathrm{mM}$ cysteamine-thiol solution in 0.1 M MES buffer, rinsed in Ultrapure water, ethanol and dried 
with $\mathrm{N}_{2}$. Two equally prepared thiol-coated surfaces were then mounted face to face into the cell of the Microslit Electrokinetic Setup $(\mathrm{MES})^{24}$ to form the rectangular streaming channel. In order to obtain sample surfaces parallelism, the glass blocks were aligned under a light microscope. $\mathrm{Ag} / \mathrm{AgCl}$ electrodes, positioned at the inlet and outlet of the channel, provided the streaming current measurements at varied pressure differences applied to the electrolyte across the slit, ranging from 50 mbar to 200 mbar in both directions. The width of the slit channel was adjusted to $30 \pm 1 \mu \mathrm{m}$ by liquid flow measurement. In MES experiments, the ionic strength was tuned with $\mathrm{KCl}$ electrolyte solution to preserve the $\mathrm{Ag} / \mathrm{AgCl}$ electrodes from dissolution. $\mathrm{pH}$ adjustment and ionic strength modulation were achieved by automatic titrator filled with $\mathrm{HCl} 0.1 \mathrm{M}, \mathrm{KOH} 0.1 \mathrm{M}$ and $\mathrm{KCl} 0.1 \mathrm{M}$. All solutions used in the electrokinetic experiments were degassed under vacuum prior to use.

Preparation of cysteamine surfaces and dendrimers-decorated tips. Cysteamine-coated surfaces were prepared as follows: silicon wafers were coated by electron beam thermal evaporation with a $5 \mathrm{~nm}$-thick $\mathrm{Cr}$ layer followed by a $30 \mathrm{~nm}$-thick Au layer. Gold surfaces were cleaned for 15 minutes by UV-ozone treatment, rinsed with ethanol and dried with $\mathrm{N}_{2}$. They were then immersed for 1 hour in a $20 \mathrm{mM}$ cysteamine-thiol solution in $0.1 \mathrm{M}$ MES buffer and rinsed twice with $\mathrm{KNO}_{3}$ solution.

PAMAM-COOH dendrimers were attached in a covalent manner to the AFM tips. Oxidesharpened microfabricated $\mathrm{Si}_{3} \mathrm{~N}_{4}$ cantilevers (MSCT, Bruker corporation, Santa Barbara) were used and their spring constants (of nominal values $0.03 \mathrm{~N} \mathrm{~m}^{-1}$ ) were accurately determined on the basis of the thermal noise method. The tips were first functionalized by amine groups. To this end, the cantilevers were washed $10 \mathrm{~min}$ in Piranha solution (3:1 mixture of concentrated $\mathrm{H}_{2} \mathrm{SO}_{4}$ and $30 \% \mathrm{H}_{2} \mathrm{O}_{2}$ solution), rinsed thoroughly with ultrapure water, dried 
with $\mathrm{N}_{2}$, then rinsed 3 times with chloroform and twice with ethanol, dried and placed in an UV-ozone cleaner for $15 \mathrm{~min}$. Cleaned cantilevers were then immersed overnight into an ethanolamine solution ( $3.3 \mathrm{~g}$ of ethanolamine into $6 \mathrm{~mL}$ of DMSO), then washed three times with DMSO and twice with ethanol, and finally dried with $\mathrm{N}_{2}$.

The dendrimers were then covalently attached to the tip via $\mathrm{N}$-hydroxysuccinimide (NHS) 1ethyl-3-(3-dimethylaminopropyl)-carbodiimide (EDC) surface chemistry. Dendrimers were immersed in NHS-EDC solution at a concentration allowing 10 out of the 2048 carboxylic surface groups of a dendrimer particle to be activated. For reaching such activation level, 50 $\mu \mathrm{L}$ of the dendrimer solution received from the provider $\left(1.06 \times 10^{-4} \mathrm{~mol} \mathrm{~L}^{-1}\right)$ were diluted in $2 \mathrm{~mL}$ ultrapure water containing EDC at $5 \mathrm{mg} \mathrm{L}^{-1}$ and $\mathrm{NHS}$ at $5.6 \mathrm{mg} \mathrm{L}^{-1}$. The amine-decorated tips were then immediately immersed in the activated-dendrimer solution, let for 1 hour, rinsed in ultrapure water and stored in water until use.

AFM force measurements. AFM force measurements were performed at room temperature using an Asylum MFP-3D atomic force microscope (Santa Barbara,CA, USA) with IGOR Pro 6.04 operation software (Wavemetrics, Lake Osewego, OR, USA). Force-separation distance curves for interacting cysteamine-coated surfaces and dendrimer-decorated tips were obtained in $\mathrm{KNO}_{3}$ solution. For consistency, the same decorated tip was used for all measurements at one $\mathrm{pH}$ condition, and the background electrolyte (of controlled $\mathrm{pH}$ ) was sequentially replaced by a more concentrated one. The force reversal upon increasing monovalent electrolyte concentration was obtained in triplicate. For each $\mathrm{pH}$ and $\mathrm{KNO}_{3}$ concentration condition tested, multiple $(16 \times 16)$ force-distance curves were recorded on 5 $\mu \mathrm{m} \times 5 \mu \mathrm{m}$ areas on the cysteamine support. Unless otherwise stated, all force curves were 
obtained using a maximum applied force of $500 \mathrm{pN}$, and approach and retraction speeds of $500 \mathrm{~nm} \mathrm{~s}^{-1}$.

\section{FIGURE CAPTIONS}

Fig. 1. Schematics of the dendrimer hyperbranched structure and of experimental set-ups.

a) Carboxylate-terminated poly(amidoamine) (PAMAM) dendrimers of generation 8.5 consisting of secondary and protonable tertiary amine groups located at the branching points of the particle core (green circles) and dissociable carboxylic groups that terminate each branch of the structure (orange circles). b) Electrostatic interactions between an AFM tip decorated by PAMAM-COOH dendrimers and the SAM surface are assessed via AFM force measurements performed in a monovalent background electrolyte $\left(\mathrm{KNO}_{3}\right)$ at various concentrations and solution $\mathrm{pH}$ values. c) Streaming current channel for electrokinetic measurements on $\mathrm{NH}_{2}$-terminated self-assembled-monolayer (SAM) surfaces . The streaming current is generated by applying a pressure gradient $\Delta P$ in a microchannel of section $S$ and length $L$. Schematics are not to scale.

Fig. 2. Electrokinetic properties of PAMAM-COOH dendrimer particles and SAM surface. a) Electrophoretic mobility of G8.5 PAMAM-COOH dendrimers as a function of $\mathrm{KNO}_{3}$ electrolyte concentration at several $\mathrm{pH}$ values (indicated). Experimental data are represented by points, whereas dotted and solid lines correspond to theoretical modeling along the lines detailed in the text. b) Dependence of the (dimensionless) electrostatic surface potential of PAMAM$\mathrm{COOH}$ dendrimer particles on $\mathrm{KNO}_{3}$ electrolyte concentration at several $\mathrm{pH}$ values (indicated) as derived from modeling of the dendrimer electrokinetic response displayed in panel a. Model parameters used for modeling electrokinetics of G8.5 PAMAM-COOH dendrimer (see further details in Ref. $^{14}$ ): volume density of carboxylic groups in the 
peripheral surface layer $=3.7 \mathrm{M}$ (fitted value), volume density of amine groups in the particle core $=12.7 \mathrm{M}$ (evaluated from dendrimer size and known number of amine groups); Dissociation $\mathrm{pK}$ values for amines and carboxylic groups are 7.4 and 3.7, respectively (fitted values); radius of the core where amines are viewed as homogeneously distributed point charges $=4 \mathrm{~nm}$ (known from dendrimer structure), thickness of the surface layer where carboxylic groups are viewed as homogeneously distributed point charges $=0.54 \mathrm{~nm}$ (known from dendrimer structure). c) Dependence of the streaming current normalized by the applied pressure gradient on $\mathrm{KCl}$ concentration at $\mathrm{pH} 3.5$ and 5.1 for the SAM surface and corresponding (dimensionless) zeta-potential (inset) estimated with use of Smoluchowski equation (eq 1$)$.

Fig. 3. Dendrimer/SAM electrostatic interactions switch from repulsion to attraction with increasing the concentration of background monovalent electrolyte. a) Point of zero mobility (PZM) of G8.5 PAMAM-COOH dendrimer versus $\mathrm{KNO}_{3}$ electrolyte concentration. Points correspond to experimental data and solid curve to theoretical electrokinetic modeling (see Fig. 2a). b) Representative force-distance curves between dendrimer-grafted AFM tip and SAM surface measured at $\mathrm{pH} 4.7$ in background monovalent electrolyte the concentration of which is below ( $2 \mathrm{mM}$ and $10 \mathrm{mM})$, close to $(30 \mathrm{mM})$ and above $(200 \mathrm{mM})$ the PZM of the dendrimer at $\mathrm{pH}$ 4.7. The force is here divided by the effective particle radius $R_{\text {eff }}=19 \mathrm{~nm}$. c) Schematic representation of the dendrimer-decorated AFM tip approaching the SAM substrate under repulsion ( $2 \mathrm{mM}$ and $\left.10 \mathrm{mM} \mathrm{KNO}_{3}\right)$, neutral interaction $(30 \mathrm{mM})$ and attraction (200 $\mathrm{mM})$ conditions.

Fig. 4. Number of repulsive and attractive events detected versus $\mathrm{pH}$ and concentration of the monovalent electrolyte. a-c) Frequency of occurrence of repulsive (black) and attractive 
(green) force curves at $\mathrm{pH} 3.5$ (a), $\mathrm{pH} 4.7$ (b) and $\mathrm{pH} 6.5$ (c) as a function of $\mathrm{KNO}_{3}$ concentration. Each bar in the histograms refers to analysis of 256 force-distance curves measured in force-volume mode on a $5 \mu \mathrm{m} \times 5 \mu \mathrm{m}$ SAM surface area. Force measurements where distinction between attraction and repulsion was not possible (see neutral interaction on Fig. 3c) are not accounted.

Fig. 5. Detailed analysis of the force-separation distance curves. a,c) Typical approach force-distance curves under $\mathrm{pH}$ and $\mathrm{KNO}_{3}$ concentrations leading to repulsion (panel a, $\mathrm{pH}=$ 3.5, $1 \mathrm{mM} \mathrm{KNO}_{3}$ ) and attraction (panel $\mathrm{c}, \mathrm{pH}=6.5,1 \mathrm{mM} \mathrm{KNO}_{3}$ ). The inset of panel a shows a zoom of the interaction force fitted with an exponential decay function (eq. 2, red line) from which the prefactor $A$ (panel e) and the electrical Debye length $\left(\kappa^{-1}\right.$, panel b) are estimated. The so-obtained $\kappa^{-1}$ values are reported as a function of $\mathrm{KNO}_{3}$ concentration (in logarithmic scale) together with the $-1 / 2$ slope expected from theory (solid line). d) Attraction force (defined in the inset of panel c) as a function of $\mathrm{KNO}_{3}$ concentration measured upon approach of the dendrimer-grafted tip to the cysteamine-coated gold surface. e) Values of the prefactor $A$ evaluated from experiments (eq. 2) at pH 3.5 (symbols) and evaluated according to theory based on the Surface Element Integration (SEI) method (dotted lines) applied to the sphere-plan interaction geometry using an effective particle radius $R_{\text {eff }}=19 \mathrm{~nm}$ and $R_{\text {eff }}=4.5 \mathrm{~nm}$ (see text for details). f) Schematic representation of equipotential surfaces (dotted lines) in the vicinity of an AFM tip decorated with G8.5 dendrimers at sufficiently low (left) and large (right) concentrations in monovalent background electrolyte. The equipotential surfaces follow the contour of a hemisphere of equivalent radius $19 \mathrm{~nm}$ and a sphere of $4.5 \mathrm{~nm}$ in the thin and thick electric double layer regimes. 
Author contributions. A.B., C.C., I.B., R.Z. and J.F.L.D. performed the research and analysed the data; A.B. and J.F.L.D. designed the research and wrote the paper.

Acknowledgements. This work was supported by the French national programme EC2CO-Ecodyn/MicrobiEn (TOXSCALE project) and by the CNRS national programme PEPSFaiDoRA (NanodUP project). Sylvie Derclaye from the University catholic of Louvain is acknowledged for gold surface deposition and Jalal Bacharouche for his help related to the use of the AFM software. We thank the Laboratoire de Chimie Physique et Microbiologie pour l'Environnement (LCPME) for the access to the AFM facilities.

\section{References}

1. J. F. L. Duval and F. Gaboriaud, Curr. Opin. Colloid Interface Sci., 2010, 15, 184-195.

2. J. F. L. Duval, C. Werner and R. Zimmermann, Curr. Opin. Colloid Interface Sci., 2016, 24, 1-12.

3. J. R. S. Martin, I. Bihannic, C. Santos, J. P. S. Farinha, B. Demé, F. A. M. Leermakers, J. P. Pinheiro, E. Rotureau and J. F. L. Duval, Langmuir, 2015, 31, 4779-4790.

4. R. Zimmermann, S. S. Dukhin, C. Werner and J. F. L. Duval, Curr. Opin. Colloid Interface Sci., 2013, 18, 83-92.

5. R. Zimmermann, D. Romeis, I. Bihannic, M. C. Stuart, J. U. Sommer, C. Werner and J. F. L. Duval, Soft Matter, 2014, 10, 7804-7809.

6. J. F. L. Duval, D. Kuttner, C. Werner and R. Zimmermann, Langmuir, 2011, 27, 10739-10752.

7. J. Langlet, F. Gaboriaud, C. Gantzer and J. F. L. Duval, Biophysical Journal, 2008, 94, 32933312.

8. S. Raafatnia, O. A. Hickey and C. Holm, Phys. Rev. Lett, 2014, 113.

9. S. S. Dukhin, R. Zimmermann, J. F. L. Duval and C. Werner, J. Colloid Interface Sci., 2010, 350,

$1-4$.

10. T. Shinagawa, H. Ohshima and T. Kondo, Biophys. Chem., 1992, 43, 149-156.

11. J. Lyklema, Colloids Surf. A Physicochem. Eng. Asp., 2006, 291, 3-12.

12. S. Pianegonda, M. C. Barbosa and Y. Levin, Europhys Lett., 2005, 71, 831-837.

13. I. Semenov, S. Raafatnia, M. Sega, V. Lobaskin, C. Holm and F. Kremer, Phys. Rev. E, 2013, 87.

14. M. Moussa, C. Caillet, R. M. Town and J. F. L. Duval, Langmuir, 2015, 31, 5656-5666.

15. A. M. Caminade, A. Ouali, R. Laurent, C. O. Turrin and J. P. Majoral, Coord. Chem. Rev., 2016, 308, 478-497.

16. W. W. Gao, S. Thamphiwatana, P. Angsantikul and L. F. Zhang, Wiley Interdiscip. Rev. Nanomed. Nanobiotechnol., 2014, 6, 532-547.

17. B. Noriega-Luna, L. A. Godinez, F. J. Rodriguez, A. Rodriguez, G. Z. L. de Larrea, C. F. SosaFerreyra, R. F. Mercado-Curiel, J. Manriquez and E. Bustos, J. Nanomater., 2014.

18. M. Selin, L. Peltonen, J. Hirvonen and L. M. Bimbo, J. Drug Deliv. Sci., 2016, 34, 10-20.

19. R. Pericet-Camara, G. Papastavrou and M. Borkovec, Macromolecules, 2009, 42, 1749-1758. 
20. M. Finessi, I. Szilagyi and P. Maroni, J. Colloid Interface Sci., 2014, 417, 346-355.

21. I. Popa, G. Papastavrou and M. Borkovec, Phys. Chem. Chem. Phys., 2010, 12, 4863-4871.

22. Y. H. Niu, L. Sun and R. A. Crooks, Macromolecules, 2003, 36, 5725-5731.

23. U. Boas, J. B. Christensen and P. M. H. Heegaard, Journal of Materials Chemistry, 2006, 16, 3786-3798.

24. R. Schweiss, P. B. Welzel, C. Werner and W. Knoll, Colloids Surf. A Physicochem. Eng. Asp., 2001, 195, 97-102.

25. J. Lyklema, In Fundamentals of Interface and Colloid Science, ed. Academic Press, 2005, Vol. IV Pair Interactions, 3.

26. M. Giesbers, J. M. Kleijn and M. A. C. Stuart, J. Colloid Interface Sci., 2002, 252, 138-148.

27. S. Bhattacharjee and M. Elimelech, J. Colloid Interface Sci., 1997, 193, 273-285.

28. B. V. Derjaguin, Kolloid Z., 1934, 69, 155.

29. U. Ascher, J. Christiansen and R. D. Russell, ACM Trans. Math. Software, 1981, 7, 209-222.

30. W. H. Press, S. A. Teukolsky, W. T. Vetterling and B. P. Flannery, In Numerical recipes : The art of Scientific computing, ed. Cambridge University Press, New York, 1985.

31. D. A. Tomalia and J. M. J. Frechet, J. Polym. Sci. A Polym. Chem., 2002, 40, 2719-2728. 